\title{
Stacked Central Configurations for the Spatial Nine-Body Problem
}

\author{
Su Xia and Deng Chunhua \\ Faculty of Mathematics and Physics, Huaiyin Institute of Technology, Huaian 223003, China \\ Correspondence should be addressed to Su Xia; dchsx2005@163.com
}

Received 25 April 2013; Accepted 3 June 2013

Academic Editors: Y.-S. Piao and M. Shibata

Copyright ( 12013 S. Xia and D. Chunhua. This is an open access article distributed under the Creative Commons Attribution License, which permits unrestricted use, distribution, and reproduction in any medium, provided the original work is properly cited.

We show the existence of the twisted stacked central configurations for the 9-body problem. More precisely, the position vectors $x_{1}, x_{2}, x_{3}, x_{4}$, and $x_{5}$ are at the vertices of a square pyramid $\Sigma$; the position vectors $x_{6}, x_{7}, x_{8}$, and $x_{9}$ are at the vertices of a square $\Pi$.

\section{Introduction and Main Results}

The classical $n$-body problem $[1,2]$ concerns the motion of $n$ mass points moving in space according to Newton's law:

$$
m_{i} \ddot{x}_{i}=-\sum_{j=1, j \neq i}^{n} \frac{m_{i} m_{j}\left(x_{i}-x_{j}\right)}{r_{i j}^{3}}, \quad i=1,2, \ldots, n .
$$

Here, $x_{i} \in \mathbb{R}^{d}$ is the position of mass $m_{i}>0$, the gravitational constant is taken equal to 1 , and $r_{i j}=\left|x_{i}-x_{j}\right|$ is the Euclidean distance between $x_{i}$ and $x_{j}$.

The space of configuration is defined by

$$
X=\left\{\left(x_{1}, \ldots, x_{n}\right) \in\left(\mathbb{R}^{d}\right)^{n}: x_{i} \neq x_{j} \forall i \neq j\right\},
$$

while the center of mass is given by

$$
c=\frac{m_{1} x_{1}+\cdots+m_{n} x_{n}}{M}
$$

where $M=m_{1}+\cdots+m_{n}$ is the total mass.

A configuration $x=\left(x_{1}, \ldots, x_{n}\right) \in X$ is called a central configuration $[2,3]$ if there exists a constant $\lambda$, called the multiplier, such that

$$
-\lambda\left(x_{i}-c\right)=\sum_{j=1, j \neq i}^{n} \frac{m_{j}\left(x_{j}-x_{i}\right)}{r_{i j}^{3}}, \quad i=1,2, \ldots, n .
$$

It is easy to see that a central configuration remains a central configuration after a rotation in $\mathbb{R}^{d}$ and a scalar multiplication. More precisely, let $A \in \mathrm{SO}(d)$ and $a>0$, if $x=\left(x_{1}, \ldots, x_{n}\right)$ is a central configuration, so are $A x=$ $\left(A x_{1}, \ldots, A x_{n}\right)$ and $a x=\left(a x_{1}, \ldots, a x_{n}\right)$.

Two central configurations are said to be equivalent if one can be transformed to the other by a scalar multiplication and a rotation. In this paper, when we say a central configuration, we mean a class of central configurations as defined by the above equivalent relation.

Central configurations of the $n$-body problem are important because they allow the computation of homographic solutions; if the $n$ bodies are heading for a simultaneous collision, then the bodies tend to a central configuration (see $[3,4]$ ); there is a relation between central configurations and the bifurcations of the hypersurfaces of constant energy and angular momentum (see [5]).

In this paper, we are interested in spatial central configurations, that is, $d=3$. In 2005, Hampton [6] provides a new family of planar central configurations for the 5body problem with an interesting property: the central configuration has a subset of three bodies forming a central configuration of the 3-body problem. The authors [7] find new classes of central configurations of the 5-body problem which are the ones studied by Hampton [6] having three bodies in the vertices of an equilateral triangle, but the other two, instead of being located symmetrically with respect to a perpendicular bisector, are on the perpendicular bisector. The 
stacked central configurations studied by Hampton [6] were completed by Llibre et al. [8] (see also [9]).

Zhang and Zhou [10] showed the existence of double pyramidal central configurations of $N+2$-body problem. The authors [11-13] provided new examples of stacked central configurations for the spatial 7-body problem where four bodies are at the vertices of a regular tetrahedron and the other three bodies are located at the vertices of an equilateral triangle.

In this paper, we find new classes of stacked spatial central configurations for the 9-body problem which have five bodies at the vertices of a square pyramid, and the other four bodies are located at the vertices of a square. More precisely, the spatial central configurations considered here satisfy the following (see Figure 1): the position vectors $x_{1}, x_{2}, x_{3}, x_{4}$, and $x_{5}$ are at the vertices of a square pyramid $\Sigma$; the position vectors $x_{6}, x_{7}, x_{8}$, and $x_{9}$ are at the vertices of a square $\Pi$.

Without loss of generality, we can assume that

$$
\begin{array}{ccc}
x_{1}=(1,0,0), & x_{2}=(0,1,0), & x_{3}=(-1,0,0), \\
x_{4}=(0,-1,0), & x_{5}=(0,0, h), & x_{6}=(x, 0, y), \\
x_{7}=(0, x, y), & x_{8}=(-x, 0, y), & x_{9}=(0,-x, y),
\end{array}
$$

where $x>0, y \in \mathbb{R}$, and $y \neq 0$; the positive constant $h$ satisfies the equation

$$
\frac{2}{r_{15}^{3}}=\frac{1}{r_{12}^{3}}+\frac{1}{r_{13}^{3}}
$$

(see [10] and the references therein); that is, $h=1.26276522$.

The main results of this paper are the following.

Theorem 1. Consider the spatial configurations according to Figure 1, in order that the nine mass points are in a central configuration, the following statements are necessary:

(1) the masses $m_{1}, m_{2}, m_{3}$, and $m_{4}$ must be equal;

(2) the masses $m_{6}, m_{7}, m_{8}$, and $m_{9}$ must be equal.

Theorem 2. There exist points $\left(x_{0}, y_{0}\right) \in T^{-1}(0) \cap D$ (see Figure 2) such that the nine bodies take the coordinates

$$
\begin{gathered}
x_{1}=(1,0,0), \quad x_{2}=(0,1,0), \\
x_{3}=(-1,0,0), \quad x_{4}=(0,-1,0), \\
x_{5}=(0,0, h), \quad x_{6}=\left(x_{0}, 0, y_{0}\right), \\
x_{7}=\left(0, x_{0}, y_{0}\right), \quad x_{8}=\left(-x_{0}, 0, y_{0}\right), \\
x_{9}=\left(0,-x_{0}, y_{0}\right) .
\end{gathered}
$$

Then, there are positive solutions of $m_{1}, m_{5}, m_{6}$ such that these bodies form a spatial central configuration according to Figure 1.

The proofs of the theorems are given in the next sections.

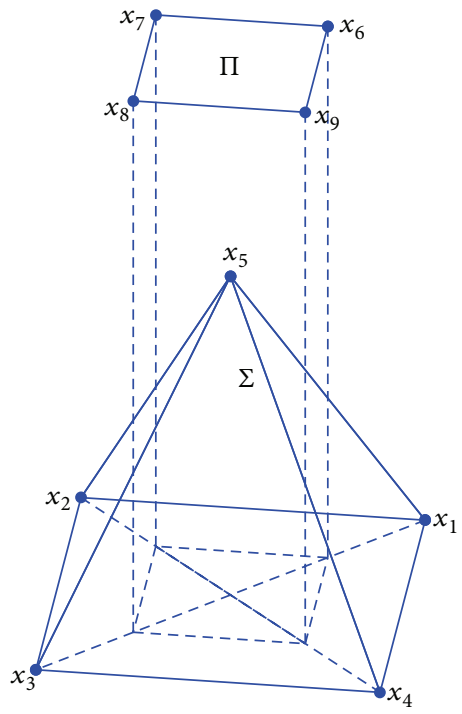

Figure 1: The configuration for the 9-body problem.

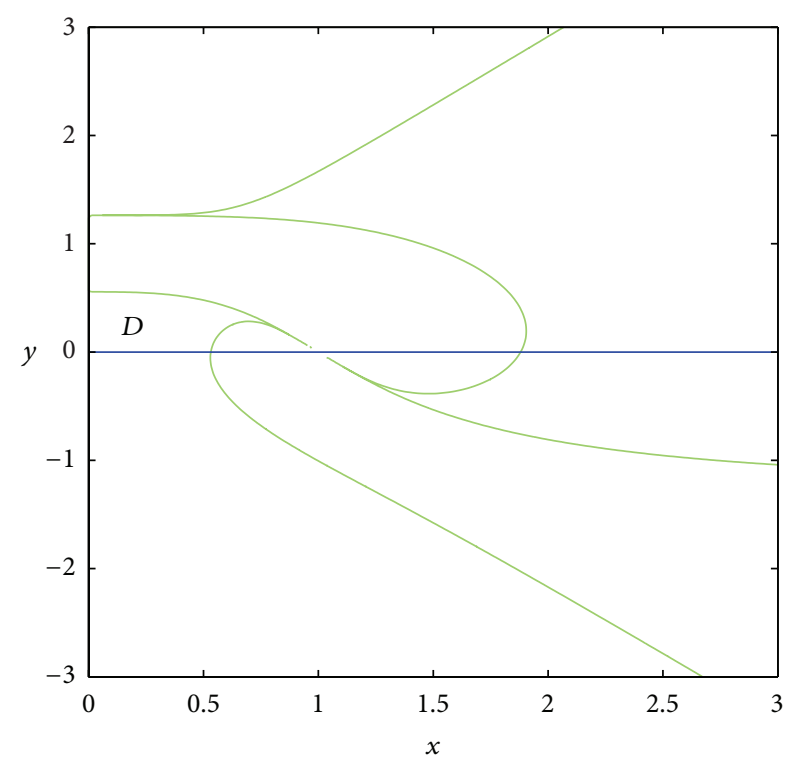

FIgURE 2: The region $D$.

\section{Proof of Theorem 1}

For the spatial central configurations, instead of working with (4), we consider the Dziobek-Laura-Andoyer equations (see $[9,11-13]$ and the references therein):

$$
f_{i j k}=\sum_{l=1, l \neq i, j, k}^{n} m_{l}\left(d_{i l}-d_{j l}\right) \Delta_{i j k l}=0
$$

for $1 \leq i<j \leq n, k=1, \ldots, n, k \neq i, j$. Here, $d_{i j}=1 / r_{i j}^{3}$ and $\Delta_{i j k l}=\left(x_{i}-x_{j}\right) \wedge\left(x_{i}-x_{k}\right) \cdot\left(x_{i}-x_{l}\right)$. Thus, $\Delta_{i j k l}$ gives six times the signed volume of the tetrahedron formed by the bodies with positions $x_{i}, x_{j}, x_{k}$, and $x_{l} ;(8)$ is a system of $n(n-1)(n-2) / 2$ equations. 
For the 9-body problem, (8) is a system of 252 equations. According to Figure 1, our class of configurations with nine bodies must satisfy

$$
\begin{aligned}
& r_{12}=r_{23}=r_{34}=r_{14}=\sqrt{2}, \quad r_{13}=r_{24}=2, \\
& r_{67}=r_{78}=r_{89}=r_{69}=\sqrt{2} x, \quad r_{68}=r_{79}=2 x, \\
& r_{16}=r_{27}=r_{38}=r_{49}=\sqrt{(x-1)^{2}+y^{2}}, \\
& r_{17}=r_{19}=r_{26}=r_{28}=r_{37}=r_{39}=r_{46} \\
& =r_{48}=\sqrt{x^{2}+1+y^{2}}, \\
& r_{18}=r_{29}=r_{36}=r_{47}=\sqrt{(x+1)^{2}+y^{2}}, \\
& r_{15}=r_{25}=r_{35}=r_{45}=\sqrt{1+h^{2}}, \\
& r_{56}=r_{57}=r_{58}=r_{59}=\sqrt{x^{2}+(y-h)^{2}} .
\end{aligned}
$$

Due to assumption (5) and the definition of $\Delta_{i j k l}$, we have several symmetries in the signed volumes.

By using the symmetries and the properties of $\Delta_{i j k l}$, we obtain the following results.

Lemma 3. In order to have a spatial central configuration according to Figure 1, a necessary condition is that the masses $m_{1}, m_{2}, m_{3}$, and $m_{4}$ must be equal.

Proof. It is sufficient to consider the equations $f_{687}=0$ and $f_{796}=0$ :

$$
\begin{aligned}
& f_{687}=\left(m_{1}-m_{3}\right)\left(d_{16}-d_{18}\right) \Delta_{6871}=0, \\
& f_{796}=\left(m_{2}-m_{4}\right)\left(d_{16}-d_{18}\right) \Delta_{7962}=0 .
\end{aligned}
$$

For our class of central configurations, we have $d_{16}-d_{18} \neq 0$, $\Delta_{6871} \neq 0$, and $\Delta_{7962} \neq 0$. So the above equations hold if and only if $m_{1}=m_{3}, m_{2}=m_{4}$. Consider the expression of $f_{678}=$ 0 :

$$
\begin{aligned}
f_{678}= & \left(m_{1}-m_{2}\right)\left(d_{16}-d_{17}\right) \Delta_{6781} \\
& +\left(m_{3}-m_{4}\right)\left(d_{18}-d_{17}\right) \Delta_{6783}=0 .
\end{aligned}
$$

Substituting $m_{1}=m_{3}, m_{2}=m_{4}$ into the above equation, we have

$$
f_{678}=\left(m_{1}-m_{2}\right)\left(d_{16}+d_{18}-2 d_{17}\right) \Delta_{6781}=0 .
$$

For our class of central configurations, we have $d_{16}+d_{18}-$ $2 d_{17} \neq 0$, since the function $g(x)=x^{-3 / 2}$ is convex for all $x>$ 0 , and $\Delta_{6781} \neq 0$. So the above equation holds if and only if $m_{1}=m_{2}$. So statement 1 of Theorem 1 is proved.

Lemma 4. If the configuration, according to Figure 1, is a central configuration, a necessary condition is that the masses $m_{6}, m_{7}, m_{8}$, and $m_{9}$ must be equal.
Proof. It is sufficient to consider the equations $f_{132}=0$ and $f_{241}=0$ :

$$
\begin{aligned}
& f_{132}=\left(m_{6}-m_{8}\right)\left(d_{16}-d_{18}\right) \Delta_{1326}=0, \\
& f_{241}=\left(m_{7}-m_{9}\right)\left(d_{16}-d_{18}\right) \Delta_{2417}=0 .
\end{aligned}
$$

For our class of central configurations, we have $d_{16}-d_{18} \neq 0$, $\Delta_{1326} \neq 0$, and $\Delta_{2417} \neq 0$. So the above equations hold if and only if $m_{6}=m_{8}, m_{7}=m_{9}$. Consider the expression of $f_{123}=$ 0 :

$$
\begin{aligned}
f_{123}= & \left(m_{6}-m_{7}\right)\left(d_{16}-d_{17}\right) \Delta_{1236} \\
& +\left(m_{8}-m_{9}\right)\left(d_{18}-d_{17}\right) \Delta_{1238}=0 .
\end{aligned}
$$

Substituting $m_{6}=m_{8}, m_{7}=m_{9}$ into the above equation, we have

$$
f_{123}=\left(m_{6}-m_{7}\right)\left(d_{16}+d_{18}-2 d_{17}\right) \Delta_{1236}=0 .
$$

For our class of central configurations, we have $d_{16}+d_{18}-$ $2 d_{17} \neq 0$, and $\Delta_{1236} \neq 0$. So the above equation holds if and only if $m_{6}=m_{7}$. Hence, statement 2 of Theorem 1 is proved.

The proof Theorem 1 is completed.

We restrict the set of admissible masses to $m_{1}=m_{2}=$ $m_{3}=m_{4}=\alpha$ and $m_{6}=m_{7}=m_{8}=m_{9}=\beta$. Substituting $m_{1}=m_{2}=m_{3}=m_{4}=\alpha$ and $m_{6}=m_{7}=m_{8}=m_{9}=\beta$ into (8), they reduce to the following 4 equations:

$$
\begin{aligned}
f_{152}= & \beta\left(\left(d_{16}+d_{17}-2 d_{56}\right) \Delta_{1526}\right. \\
& \left.+\left(d_{17}+d_{18}-2 d_{56}\right) \Delta_{1528}\right)=0 \\
f_{162}=\alpha & \left(d_{12}+d_{13}-d_{17}-d_{18}\right) \Delta_{1623} \\
& +m_{5}\left(d_{15}-d_{56}\right) \Delta_{1625} \\
+ & \beta\left(d_{17}+d_{18}-d_{67}-d_{68}\right) \Delta_{1628}=0, \\
f_{175}=\alpha & \left(\left(d_{12}-d_{16}\right) \Delta_{1752}+\left(d_{13}-d_{17}\right) \Delta_{1753}\right. \\
& \left.+\left(d_{12}-d_{18}\right) \Delta_{1754}\right) \\
+ & \beta\left(\left(d_{16}-d_{67}\right) \Delta_{1756}+\left(d_{18}-d_{67}\right) \Delta_{1758}\right. \\
& \left.+\left(d_{17}-d_{68}\right) \Delta_{1759}\right)=0, \\
& +\left(d_{15}-d_{16}\right) \Delta_{5621}+\left(d_{15}-d_{18}\right) \Delta_{5623} \\
& \left.+\left(d_{17}\right) \Delta_{5624}\right) \\
+ & \beta\left(\left(d_{56}-d_{67}\right) \Delta_{5627}+\left(d_{56}-d_{67}\right) \Delta_{5629}\right)=0 . \\
f_{562}=\alpha &
\end{aligned}
$$

If we write $f_{152}=\beta T=\beta\left(\left(d_{16}+d_{17}-2 d_{56}\right) \Delta_{1526}+\right.$ $\left.\left(d_{17}+d_{18}-2 d_{56}\right) \Delta_{1528}\right)=0$, it follows that $T=0$ in order to have central configurations. So in the following, we restrict our central configurations to the set $T^{-1}(0)$.

Lemma 5. According to one's assumptions and the set $T^{-1}(0)$, (8) is satisfied if (17) and (18) are satisfied. 


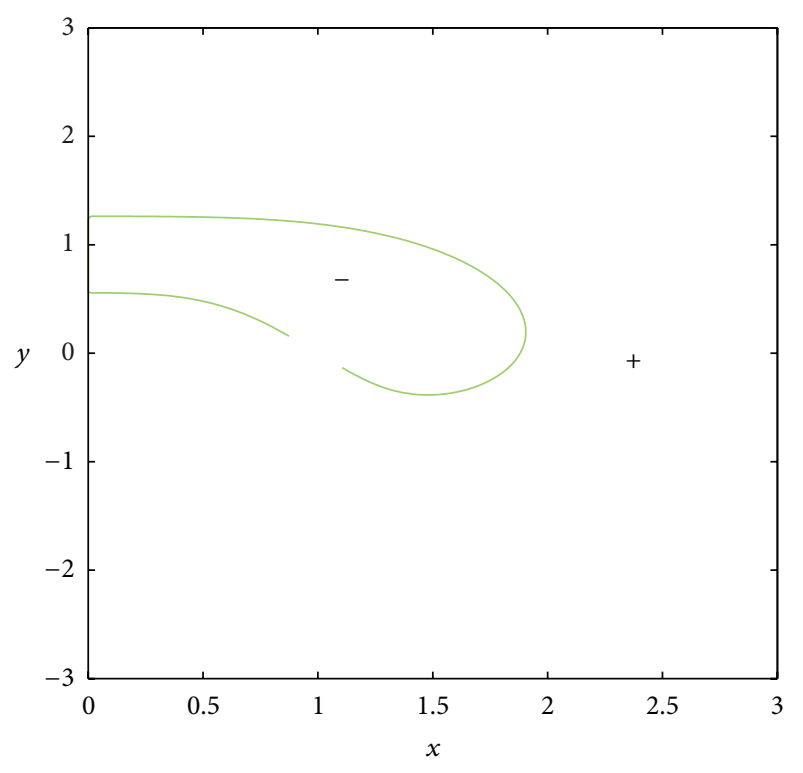

FIgURE 3: The curve $a_{21}=0$.

Proof. Under the assumptions (5), we have

$$
T=\left(d_{16}+2 d_{17}+d_{18}-4 d_{56}\right)(y-h)+h x\left(d_{16}-d_{18}\right)=0 ;
$$

that is,

$$
4(y-h) d_{56}=(y-h)\left(d_{16}+2 d_{17}+d_{18}\right)+h x\left(d_{16}-d_{18}\right) .
$$
0 .

Substituting (21) into (19), we obtain the equation $f_{175}=$

Hence in the set $T^{-1}(0), f_{175}=0$ implies $f_{562}=0$. This completes the proof.

From Lemma 5, in order to study central configurations according to Figure 1 in the set $T^{-1}(0)$, it is sufficient to study the following 2 equations:

$$
f_{162}=0, \quad f_{175}=0 .
$$

Denote by $A=\left(a_{i j}\right)$ the matrix of the coefficients of the homogeneous linear system in the variables $\alpha, m_{4}, \beta$ defined by (22). Thus,

$$
\begin{gathered}
a_{11}=\left(d_{12}+d_{13}-d_{17}-d_{18}\right) \Delta_{1623} \\
=-2 y\left(\frac{1}{2 \sqrt{2}}+\frac{1}{8}-\frac{1}{\left(x^{2}+1+y^{2}\right)^{3 / 2}}\right. \\
\left.-\frac{1}{\left((x+1)^{2}+y^{2}\right)^{3 / 2}}\right),
\end{gathered}
$$

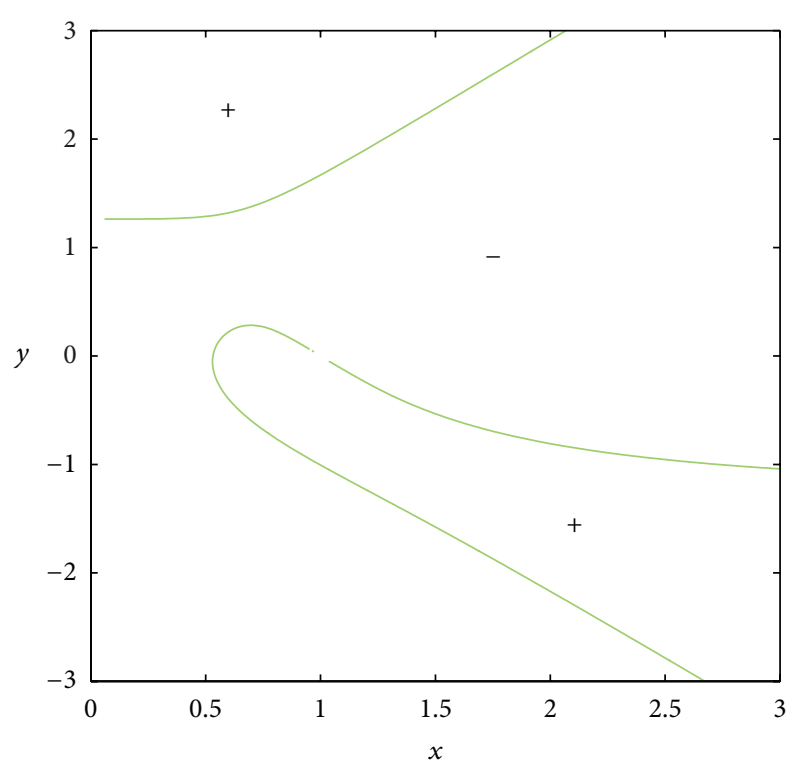

Figure 4: The curve $a_{23}=0$.

$$
\begin{aligned}
a_{12}= & \left(d_{15}-d_{56}\right) \Delta_{1625} \\
= & (-y-h x+h) \\
& \times\left(\frac{1}{\left(1+h^{2}\right)^{3 / 2}}-\frac{1}{\left(x^{2}+(y-h)^{2}\right)^{3 / 2}}\right), \\
a_{13}= & \left(d_{17}+d_{18}-d_{67}-d_{68}\right) \Delta_{1628} \\
= & -2 x y\left(\frac{1}{\left(x^{2}+1+y^{2}\right)^{3 / 2}}+\frac{1}{\left((x+1)^{2}+y^{2}\right)^{3 / 2}}\right. \\
& \left.\quad-\frac{1}{8 x^{3}}-\frac{1}{2 \sqrt{2} x^{3}}\right),
\end{aligned}
$$

$$
\begin{aligned}
a_{21}= & \left(d_{12}-d_{16}\right) \Delta_{1752}+\left(d_{13}-d_{17}\right) \Delta_{1753} \\
& +\left(d_{12}-d_{18}\right) \Delta_{1754} \\
= & (y-h)\left(\frac{1}{\left((x+1)^{2}+y^{2}\right)^{3 / 2}}\right. \\
& \left.-\frac{1}{\left((x-1)^{2}+y^{2}\right)^{3 / 2}}\right) \\
& +h x\left(\frac{1}{4}+\frac{1}{\sqrt{2}-\frac{1}{\left((x-1)^{2}+y^{2}\right)^{3 / 2}}}\right. \\
& \left.-\frac{1}{\left((x+1)^{2}+y^{2}\right)^{3 / 2}}-\frac{2}{\left(x^{2}+1+y^{2}\right)^{3 / 2}}\right),
\end{aligned}
$$




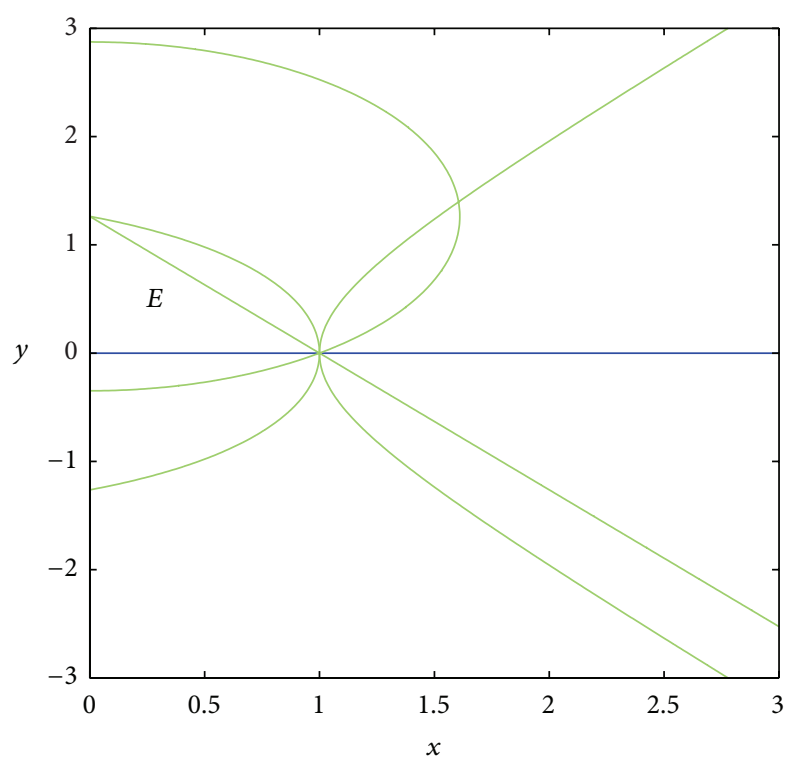

FIgURE 5: The region $E$.

$$
\begin{aligned}
a_{22}= & 0, \\
a_{23}= & \left(d_{16}-d_{67}\right) \Delta_{1756}+\left(d_{18}-d_{67}\right) \Delta_{1758} \\
& +\left(d_{17}-d_{68}\right) \Delta_{1759} \\
= & h x^{2}\left(\frac{1}{\left((x+1)^{2}+y^{2}\right)^{3 / 2}}-\frac{1}{\left((x+1)^{2}+y^{2}\right)^{3 / 2}}\right) \\
& -x(y-h) \\
& \times\left(\frac{1}{\left((x+1)^{2}+y^{2}\right)^{3 / 2}}+\frac{1}{\left((x+1)^{2}+y^{2}\right)^{3 / 2}}\right. \\
& \left.+\frac{2}{\left(x^{2}+1+y^{2}\right)^{3 / 2}}-\frac{1}{4 x^{3}}-\frac{1}{\sqrt{2} x^{3}}\right) .
\end{aligned}
$$

Let $x=\left(\begin{array}{c}\alpha \\ m_{4} \\ \beta\end{array}\right)$. Then in order to get the spatial central configuration as Figure 1, we need to find a positive solution $\alpha, m_{4}, \beta$ of the following system:

$$
A x=0,
$$

where $T=0$.

\section{The Existence of Spatial Central Configurations}

In order to prove the existence of positive solutions of (24) in the set $T^{-1}(0)$, it is sufficient to prove that the entries in each row of $A$ change the signs. So if the entries of some row of $A$ have the same signs, there are no admissible masses such that the bodies are in a central configuration according to Figure 1.

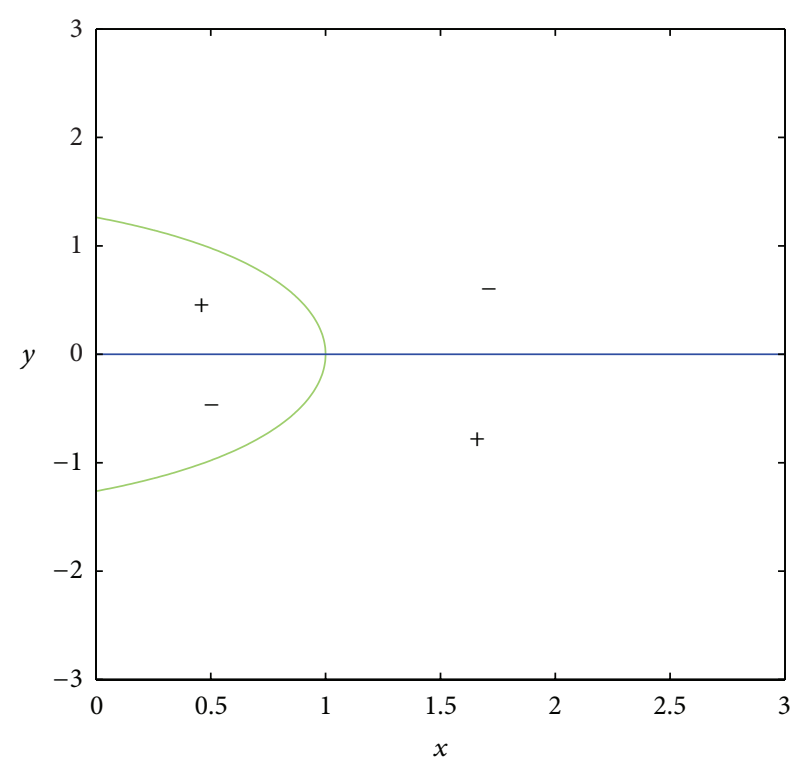

FIgURE 6: The curve $a_{11}=0$.

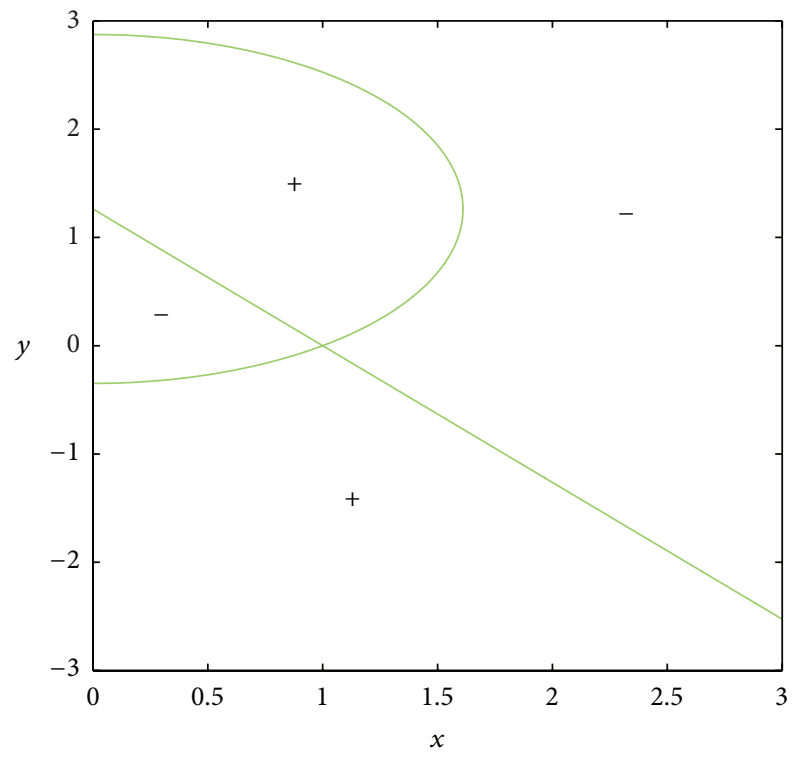

Figure 7: The curve $a_{12}=0$.

Proof of Theorem 2. Since the rank of matrix $A$ is two in the set $T^{-1}(0)$, there are nontrivial solutions of (24) in the set $T^{-1}(0)$.

Now we prove the existence of spatial central configurations according to Figure 1 for some points in the set $D$ (see Figure 2). In order to prove the existence of positive solutions of (24) in the set $T^{-1}(0)$, the entries $a_{21}, a_{23}$ of the second line in the matrix $A$ should have opposite signs. Thus, we consider the following set $D$, where $D$ is surrounded by curves $x=0$, $y=0, a_{21}=0$, and $a_{23}=0$.

In the set $D$, the entries of matrix $A$ have the following signs: $a_{21}>0, a_{23}<0$ (see Figures 3 and 4 ); $a_{11}>0, a_{12}<0$, $a_{13}>0$ because the set $D$ is included in the set $E$, where $E$ is 


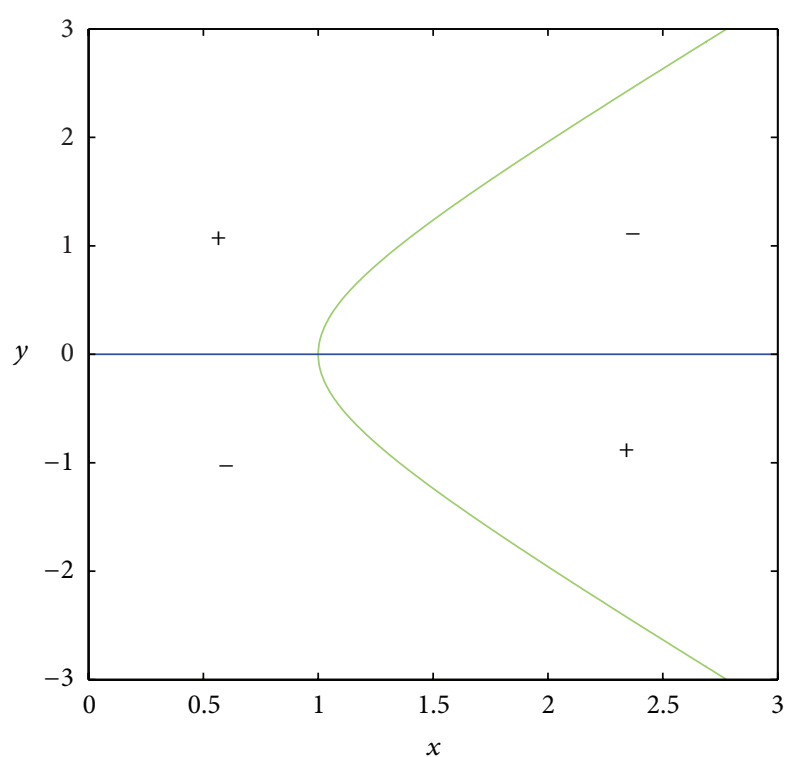

FIgURE 8: The curve $a_{13}=0$.

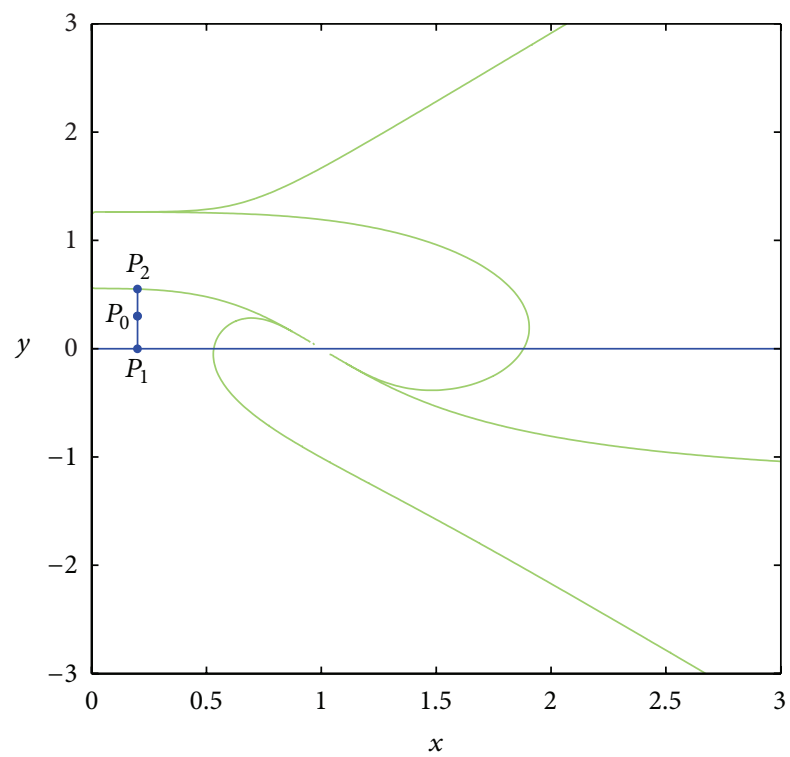

Figure 9: The existence of central configurations for the 9-body problem.

surrounded by curves $x=0, y=0$, and $y=h(1-x)$ (see Figures 5, 6, 7, and 8). In short, the signs of the entries of the matrix $A$ restricted to the set $D$ are the following:

$$
A=\left(\begin{array}{lll}
+ & - & + \\
+ & 0 & -
\end{array}\right)
$$

In the rest of the proof, we show that the set $T^{-1}(0)$ has intersection with the set $D$. We consider the subset of $D$ :

$$
L=\left\{(x, y): x=x_{1}, 0<y<y_{1}\right\}
$$

where $x_{1} \in(0,1)$. Obviously $L$ is a segment with endpoints

$$
P_{1}=\left(x_{1}, 0\right), \quad P_{2}=\left(x_{1}, y_{1}\right),
$$

(see Figure 9), and the point $\left(x_{1}, y_{1}\right)$ satisfies the equation $a_{21}=0$. Evaluating the function $T$ at these points, we have

$$
T\left(P_{1}\right)<0, \quad T\left(P_{2}\right)>0 .
$$

Thus, there exists a point $P_{0}=\left(x_{0}, y_{0}\right) \in L$, such that $T\left(P_{0}\right)=$ 0 . So at the point $P_{0}$ we have nontrivial positive solutions of (24), since the signs of the entries of the matrix $A$ at this point are the following:

$$
A\left(P_{0}\right)=\left(\begin{array}{lll}
+ & - & + \\
+ & 0 & -
\end{array}\right)
$$

Thus, the proof of Theorem 2 is completed.

\section{Acknowledgments}

The authors are supported by the Natural Science Foundation of China (NFSC11201168) and the Scientific Research Foundation of Huaiyin Institute of Technology (HGA1102).

\section{References}

[1] V. Arnold, V. Kozlov, and A. Neishtadt, Dynamical Systems, Mathematical Aspects of Classical and Celestial Machanics, Springer, Berlin, Germany, 1988.

[2] A. Wintner, Analytical Foundations of Celestial Mechanics, Princeton University Press, Princeton, NJ, USA, 1941.

[3] R. Moeckel, "On central configurations," Mathematische Zeitschrift, vol. 205, no. 4, pp. 499-517, 1990.

[4] D. Sarri, "On the role and the properties of $n$-body central configurations," Celestial Mechanics, vol. 21, no. 1, pp. 9-20, 1980.

[5] S. Smale, "Topology and mechanics II: the planar $n$-body problem," Inventiones Mathematicae, vol. 11, no. 1, pp. 45-64, 1970.

[6] M. Hampton, "Stacked central configurations: new examples in the planar five-body problem," Nonlinearity, vol. 18, no. 5, pp. 2299-2304, 2005.

[7] J. Llibre and L. F. Mello, "New central configurations for the planar 5-body problem," Celestial Mechanics and Dynamical Astronomy, vol. 100, no. 2, pp. 141-149, 2008.

[8] J. Llibre, L. F. Mello, and E. Perez-Chavela, "New stacked central configurations for the planar 5-body problem," Celestial Mechanics and Dynamical Astronomy, vol. 110, no. 1, pp. 43-52, 2011.

[9] L. F. Mello, F. E. Chaves, A. C. Fernandes, and B. A. Garcia, "Stacked central configurations for the spatial six-body problem," Journal of Geometry and Physics, vol. 59, no. 9, pp. 12161226, 2009.

[10] S. Q. Zhang and Q. Zhou, "Double pyramidal central configurations," Physics Letters A, vol. 281, no. 4, pp. 240-248, 2001.

[11] M. Hampton and M. Santoprete, "Seven-body central configurations: a family of central configurations in the spatial sevenbody problem," Celestial Mechanics and Dynamical Astronomy, vol. 99, no. 4, pp. 293-305, 2007. 
[12] L. F. Mello and A. C. Fernandes, "Stacked central configurations for the spatial seven-body problem," Qualitative Theory of Dynamical Systems, vol. 12, no. 1, pp. 101-114, 2013.

[13] X. Su and T. Q. An, "Twisted stacked central configurations for the spatial seven-body problem," Journal of Geometry and Physics, vol. 70, pp. 164-171, 2013. 


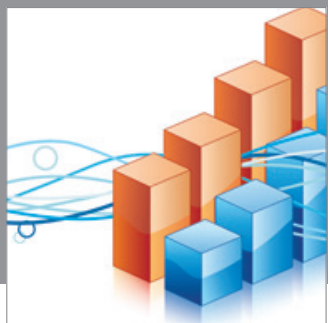

Advances in

Operations Research

mansans

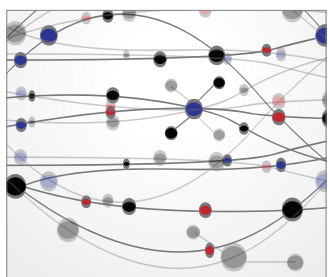

The Scientific World Journal
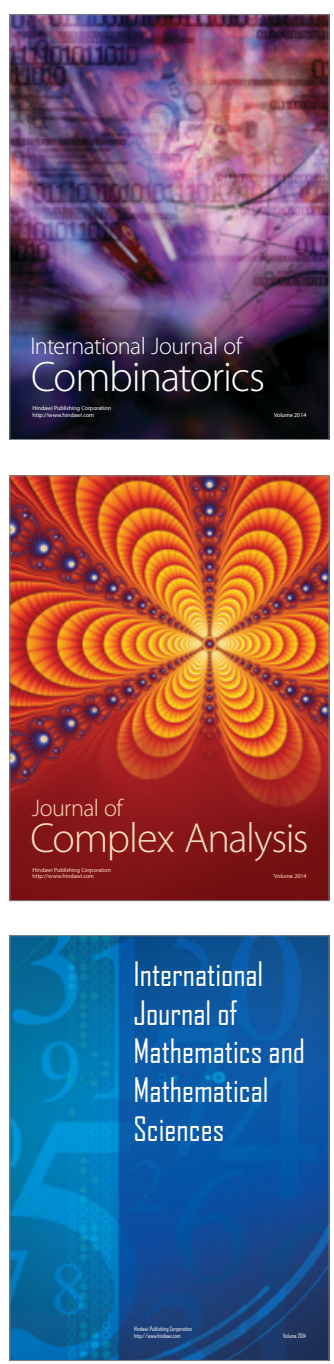
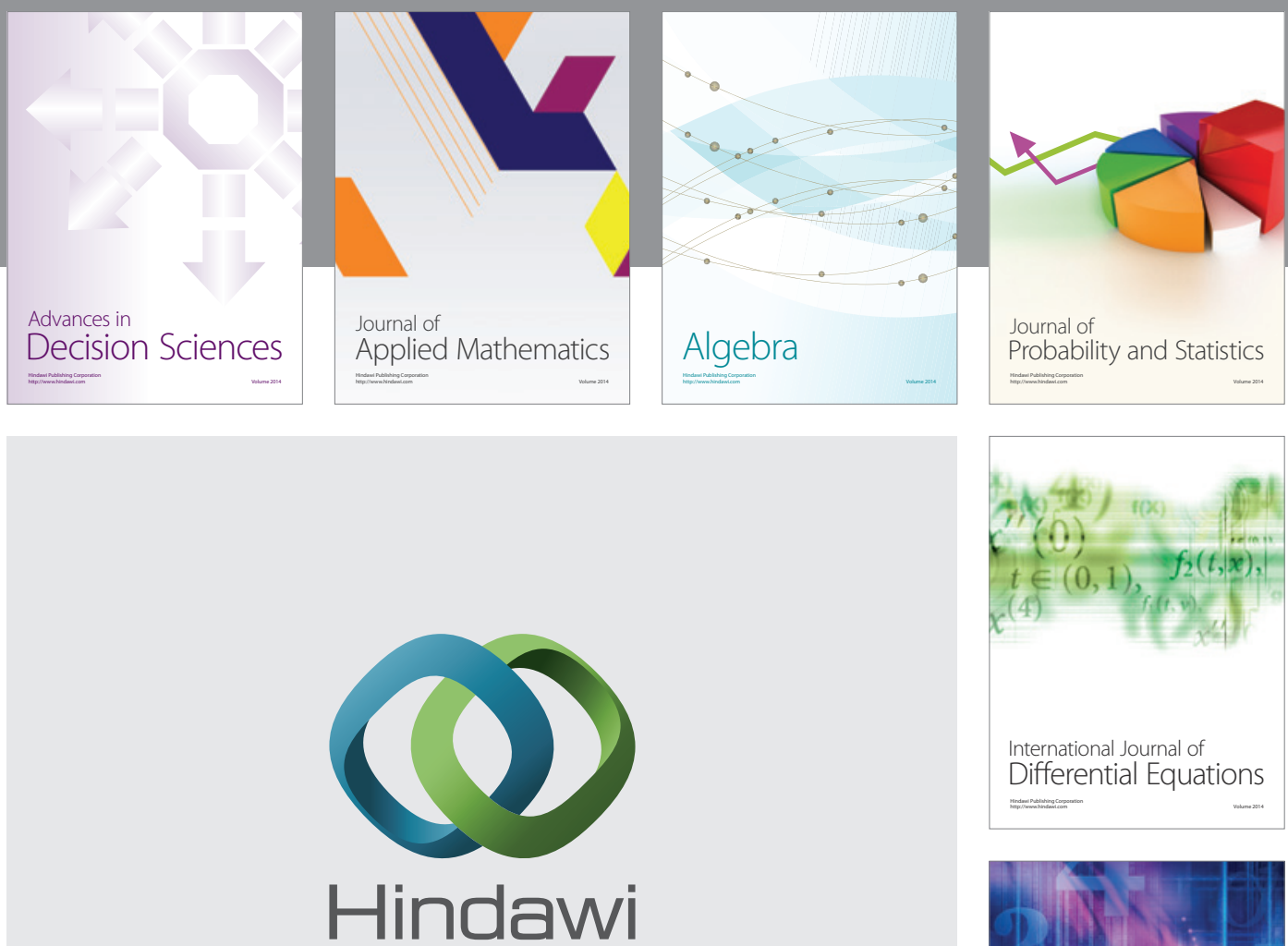

Submit your manuscripts at http://www.hindawi.com
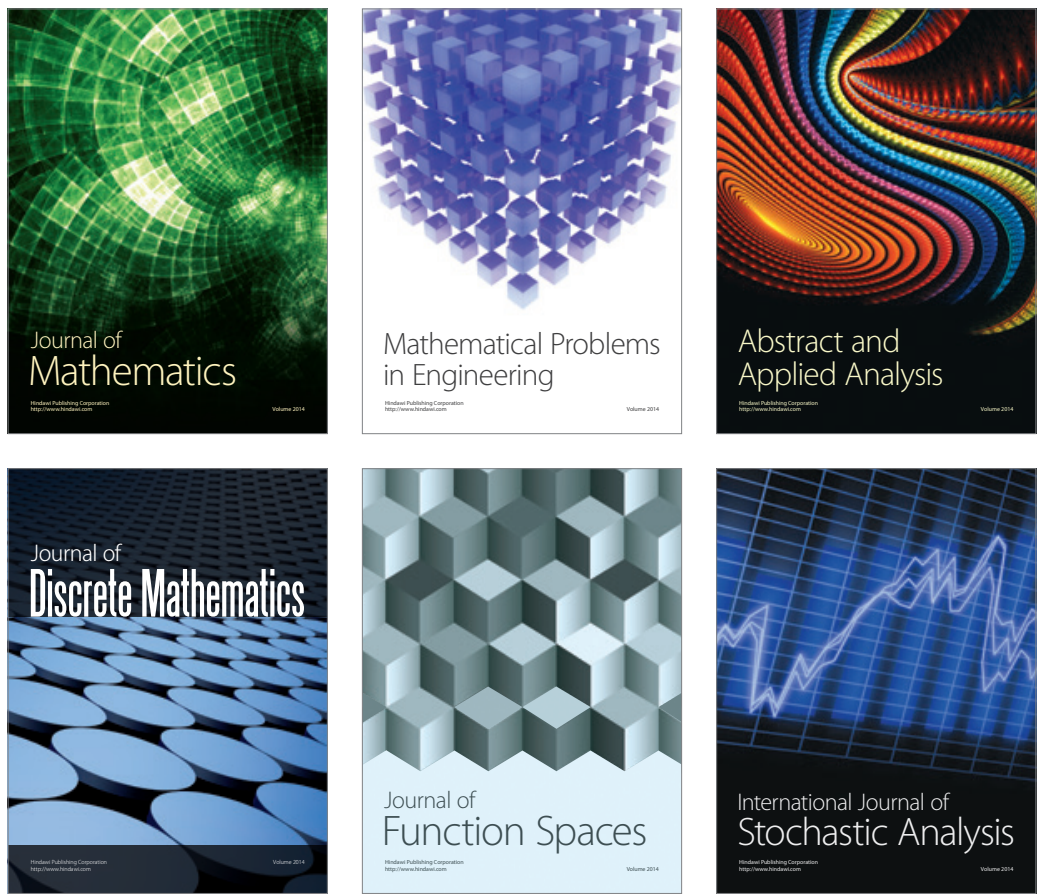

Journal of

Function Spaces

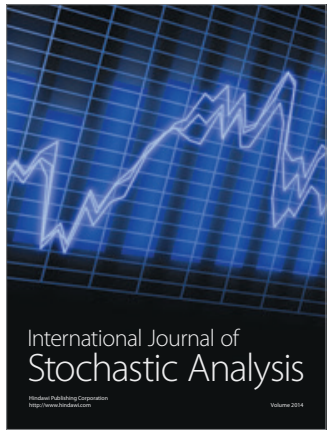

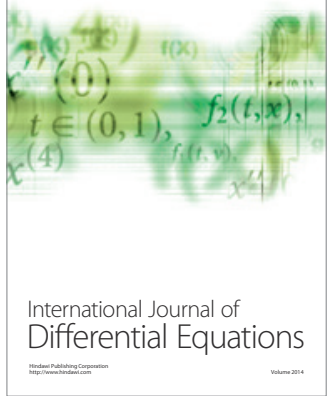
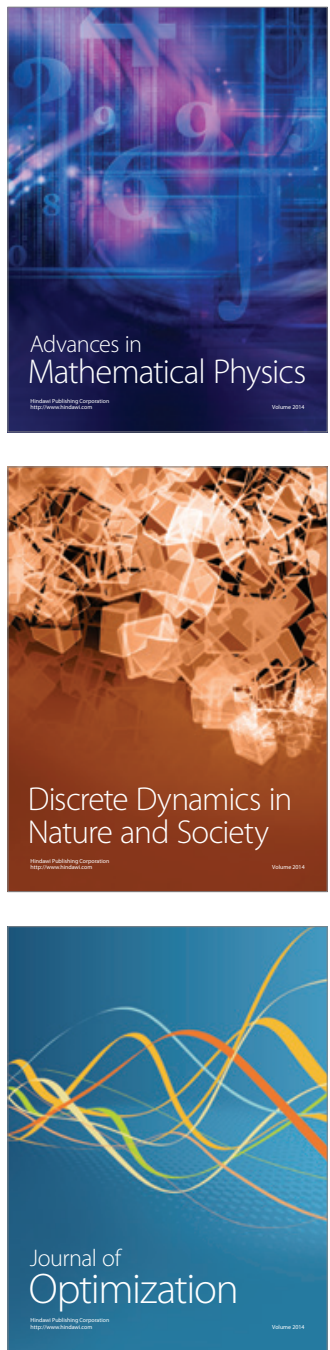